http://jmscr.igmpublication.org/home/ ISSN (e)-2347-176x ISSN (p) 2455-0450 crossref DOI: https://dx.doi.org/10.18535/jmscr/v9i5.03

Journal Of Medical Science And Clinical Research

\title{
Toxic Megacolon as a Complication of Crohns Disease: A Case Report
}

Authors

\section{Supriya Verma, V Anish Chandran, Anchana Gulati}

\begin{abstract}
Toxic megacolon is a rare and serious complication of Crohn's disease. Because of the associated high morbidity and mortality, early recognition and management of toxic megacolon is important. We had a case of Crohns disease presenting as toxic megacolon. Rarity of this complication associated with crohns disease suggest reporting of the case.

Keywords: Toxic megacolon; Crohns disease; Inflammatory bowel disease.
\end{abstract}

\section{Introduction}

Toxic megacolon (TM) is an infrequent but a potentially life-threatening complication of many colitis $^{[1]}$. Primarily associated with inflammatory bowel disease (IBD), TM is also a documented complication of severe infectious, ischemic and metabolic affections of the colon ${ }^{[2]}$. The incidence of TM in Crohn's colitis ranges from $1 \%$ to $5 \%{ }^{[3]}$. TM is characterized by total or segmental nonobstructive colonic distension of at least $6 \mathrm{~cm}$, associated with systemic toxicity ${ }^{[4]}$. The diagnosis of TM is based on criteria proposed by Jalan et al and also by Cleveland Clinical criteria. Dueto the associated high morbidity and mortality, early recognition and management of $\mathrm{TM}$ is of paramount importance.

\section{Case report}

A 40 year old patient presented with pain over the epigastrium which was acute in onset, moderate in intensity, pricking in nature and associated with 23 episodes of vomiting.USG abdomen revealed free fluid in the right iliac fossa indicating perforation. Provisional diagnosis of acute generalized peritonitis causing sigmoid perforation with grossly dilated sigmoid colon was made.

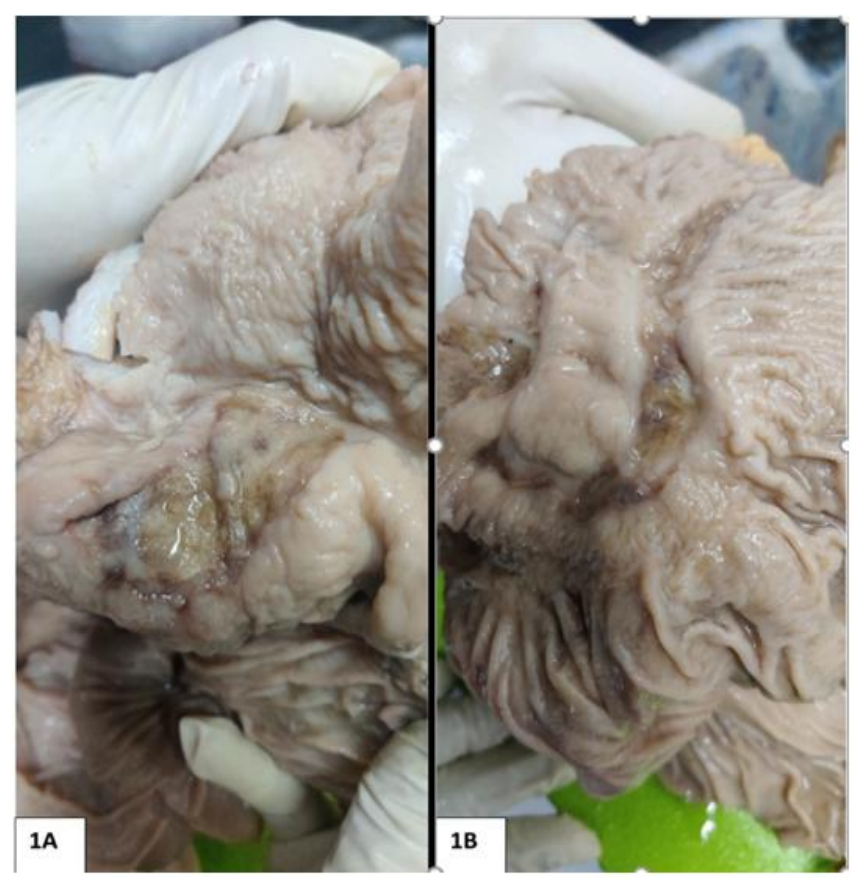

Figure 1A \& 1B: Gross photograph of intestine showing thickened wall and serpigenous ulcer with heaped up margins and dirty base 
Hartman's procedure was done (resection of dilated and perforation bearing segment of sigmoid colon with end colostomy). Blood reports revealed anemia and lymphopenia. Serum sodium and potassium levels were low. Coagulation profile was deranged.

The department of pathology at IGMC shimla received a specimen of large intestine measuring $30 \mathrm{cms}$. External surface was smooth and a perforation site measuring $8 \mathrm{~mm}$ in diameter was identified. Creeping fat was seen in external surface and the cut section revealed partly preserved mucosa with thickened areas. Rest of the mucosa showed flattening with granular appearance. Multiple serpigenous ulcers were also identified measuring $4 \mathrm{~cm}$ to $13 \mathrm{~cm}$ in length. Ulcer had heaped up margins and dirty appearing base (Figure 1A \& 1B). Multiple pseudo polyps were additionally identified.

Microscopic examination of sections from ulcer revealed apthous and knife like fissures extending through the bowel wall covered by fibrinous exudative material with underlying inflammatory granulation tissue and fibrosis (Figure 2). Transmural inflammatory infiltrate, lymphoid follicles with prominent germinal centres were noted in the sub mucosa, muscularispropria and serosa. Presence of vegetable matter was observed in the subserosal fat which confirmed perforation (Figure 5A).

Additionally pseudo polyps (Figure 5B), inflamed lamina propria, thickened muscularis mucosa, foci of misplaced epithelium in the sub mucosa identified. The submucosa showed dilated lymphatics, edema, fibrosis and neuronal hyperplasia. The muscularispropria was hypertrophied. The serosa revealed serositis, marked fibrosis, lymphoid follicles with prominent germinal centres and lobules of mature adipose tissue creeping between serosa and muscularispropria (Figure 4). Few areas revealed muscle fibers which were shortened, rounded with collection of eosinophilic cytoplasm with edema separating them, being replaced by fibrosis.
Occasional epithelioid cell granulomas were seen in the serosa (Figure 3) with mixed inflammatory cell infiltrate of neutrophils, eosinophils, lymphocytes and plasma cells.

So based on the various histological findings, a diagnosis of Megacolon secondary to Crohns disease was made.

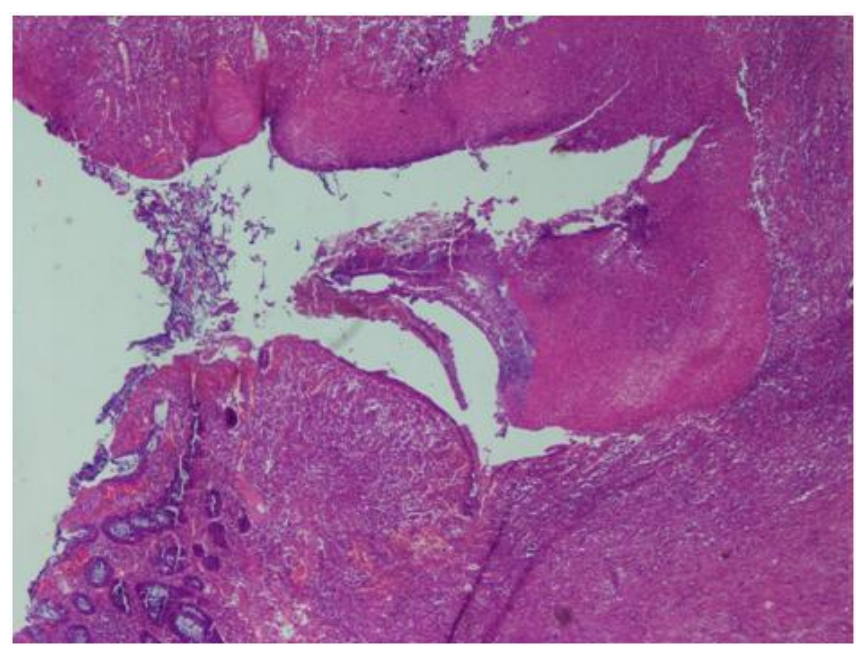

Figure 2: Microphotograph showing aphthous and knife like fissures extending into the bowel wall covered by fibrinous exudate with underlying granulation tissue (H\&E, 40x)

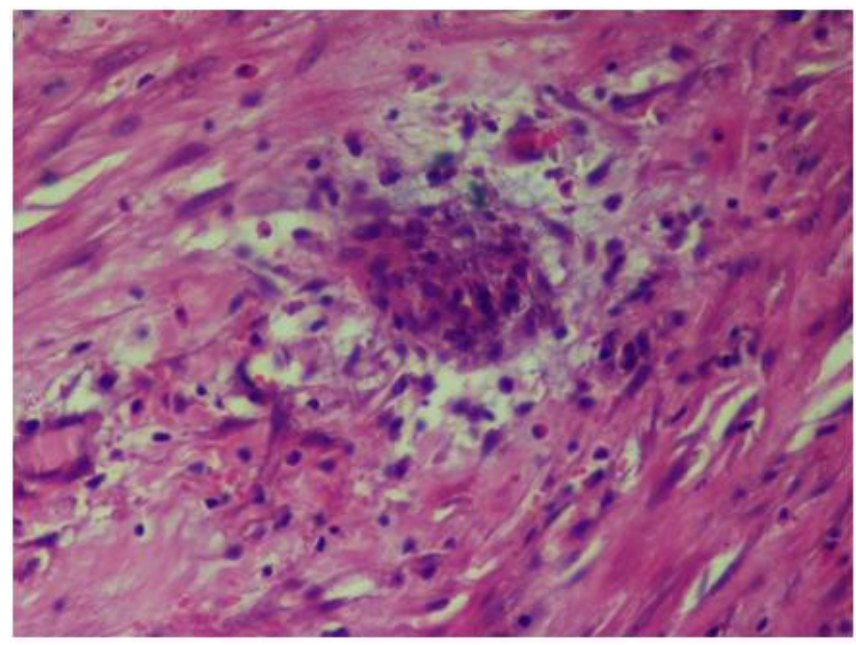

Figure 3: Microphotograph showing foci of epithelioid cell granulomas (H\&E, 40x) 


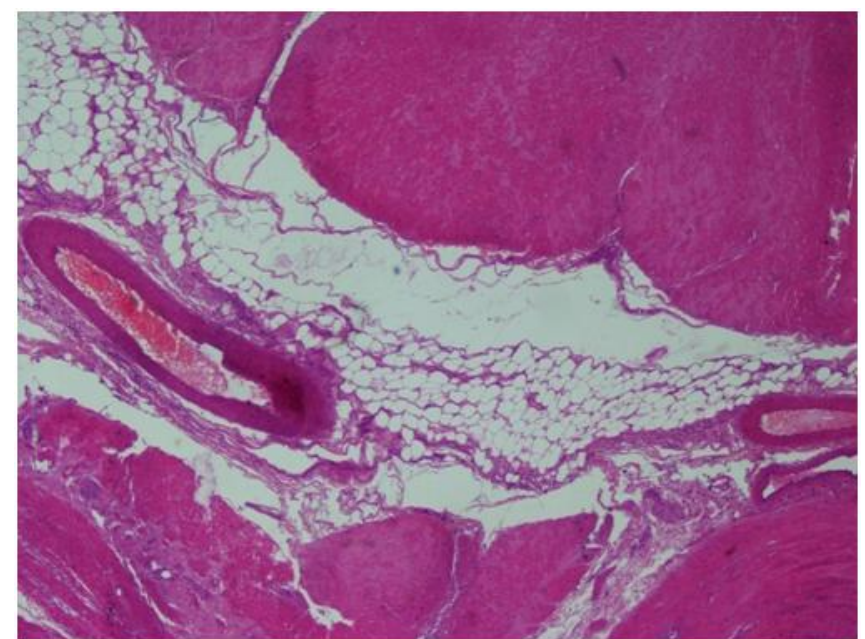

Figure 4: Microphotograph showing lobules of mature adipose tissue creeping between serosa and muscularispropria (creeping fat) (H\&E, 40x)

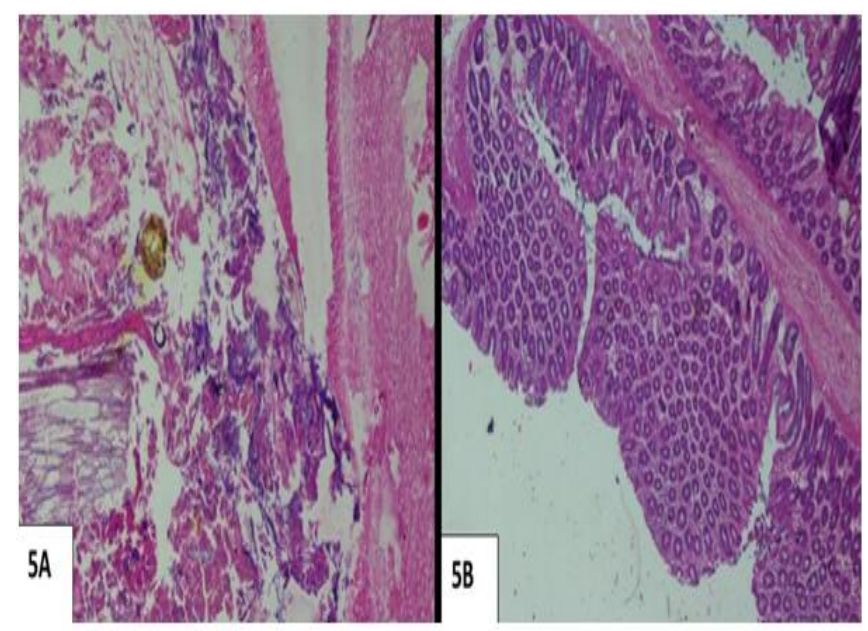

Figure 5A: Microphotograph showing vegetable matter in subserosal fat confirming perforation (H\&E, 10x)

Figure 5B: Microphotograph showing pseudopolyps (H\&E, 10x)

\section{Discussion}

Crohn's Disease (CD) is a chronic relapsing inflammatory disorder in the spectrum of inflammatory bowel disease. It can affect any part of the GIT. The clinical presentation can overlap with other inflammatory conditions like tuberculosis, ulcerative colitis, etc. To make a diagnosis of this disease, various modalities are available. Conservative treatment is the mainstay but complications like intestinal obstruction, perforations, abscess and fistula formations may require surgical intervention ${ }^{[5]}$.
It is characterized by a transmural granulomatous inflammation which can affect any part of the gastrointestinal tract, most commonly the ileum, colon or both. India is projected to have one of the highest disease burden of IBD across the globe. IBD in the West is characterized by a bimodal age distribution with peaks at age groups 20-39 and 60-79 years. In a country-wide survey of IBD from India, the mean age at diagnosis of ulcerative colitis (UC) and CD was 38.5 and 35.9 years, respectively, and was not associated with a second peak ${ }^{[5]}$. Chronic diarrhoea, abdominal pain , weight loss and blood, mucus or both in stools are the most common presenting symptoms in CD. Extra intestinal manifestations affect approximately a third of patients with IBD. The most commonly observed extra intestinal manifestations are primary peripheral arthritis, aphthous stomatitis, uveitis, erythema nodosum and ankylosing spondylitis. The diagnoses of Crohn's disease and ulcerative colitis are made on clinical grounds supplemented with objective findings of radiological, endoscopic, and histological examination ${ }^{[6]}$. Ultrasonography, contrast enhanced ultrasonography, multi detector CT enteroclysis, MRI enteroclysis and 99mTcHMPAO-labeled leukocyte scintigraphy are the various radiological aids which help in diagnosing CD. Typical endoscopic findings in CD include discontinuous distribution of longitudinal ulcers (defined as $\geq 4$ to $5 \mathrm{~cm}$ ulcers in the Japanese criteria), cobblestone appearance, and/or small aphthous ulcerations arranged in a longitudinal fashion $^{[7]}$.

$\mathrm{TM}$ is a rare and lethal complication of CD. TM affects male and female patients of all ages ${ }^{[4]}$. The incidence of TM in CD ranges from $1 \%$ to $5 \%$ [3].

Toxic colitis is a life-threatening condition that is most commonly seen in patients with inflammatory bowel disease. It was first described by Marshak in 1950 as a complication of ulcerative colitis ${ }^{[1]}$. For over fifteen years it was thought to be specific to UC, until Schachter published the first case report in 1967 of toxic 
colitis in a patient with Crohn's disease ${ }^{[8]}$. The incidence of toxic colitis in adults is $1-8 \%$ in those presenting to a hospital with Crohn's disease, and 2-21\% in those presenting with ulcerative colitis ${ }^{[9]}$.

TM is more likely to complicate pancolitis than segmental disease. It occurs early in the course of CD and may be the initial disease in $20 \%$ of cases. Predisposing factors are sometimes identified.CMV infection may trigger toxic megacolon in patients with IBD. CMV infection may be responsible for an increased morbidity and mortality rate in patients with severe IBD. The most widely used criteria for the clinical diagnosis of TM, proposed by Jalan et $a l^{[10]}$ were radiographic evidence of colonic distension, at least three of the following: fever $>38^{\circ} \mathrm{C}$, heart rate $>120 / \mathrm{min}$, neutrophilic leucocytosis $>10.5 \times 10^{9} / \mathrm{L}$, anaemia $(\mathrm{Hb}<10 \mathrm{~g} / \mathrm{dl})$. In addition, to the above, at least one of the following symptoms: dehydratation, altered consciousness, electrolyte disturbances, hypotension. Midtransverse colon diameter $>5.5 \mathrm{~cm}$ on abdominal $\mathrm{X}$-ray was also thought to be predictive ${ }^{[11]}$. In 2009, the Cleveland Clinic Criteria were proposed. This was a much simpler system and did not rely on colonic dilation as a factor. The diagnosis could be made by the presence of any three of the following in the appropriate setting: 1) temperature $>38{ }^{\circ} \mathrm{C}$, 2) heart rate $>100 \mathrm{bpm}, 3$ ) hemoglobin $<10 \mathrm{~g} / \mathrm{dL}$, 4) white blood cell count $>10,500 / \mathrm{mm}^{3}$, and 5) albumin $<3 \mathrm{~g} / \mathrm{dL}^{[12]}$.

Grossly, the length of the segments involved in $\mathrm{CD}$ is variable and the lesions are separated by uninvolved skip areas. Macroscopic lesions are apparent on the mucosal and serosal side of the bowel wall. Fistulas are commonly associated with strictures and therefore the clinical distinction is often limited to two types: the perforating type and non-perforating type with predominantly mucosal lesions. The mucosa may appear normal or may show multiple small (1-2 $\mathrm{mm}$ in size) punctiform, rounded nodules or superficial erosions known as 'aphthoid lesions'. Over a period of time, the erosions become confluent and give rise to larger longitudinal ulcers, known as serpiginous ulcers. The combination of longitudinal and transverse ulceration in an edematous mucosa induces a characteristic 'cobblestone' aspect. Ulcerations are more common on the mesenteric border of the small intestine. Being a transmural disease, the bowel wall is thickened with involvement of the submucosa, the muscularispropria, the subserosa and mesenteric fat. Mesenteric fat partially surrounds the intestine, extending from the mesenteric attachment anteriorly and posteriorly corresponding to the involved segment. This phenomenon, known as 'fat wrapping', is specific for CD. Inflammatory pseudopolyps of the colon and small intestine, identical to those described in UC can be observed in CD. These are usually tall mucosal outgrowths measuring a few millimeters in length ${ }^{[13]}$.

Microscopically, foci of inflammation inbetween mostly normal mucosa consist of lymphocytes, plasma cells, neutrophils, eosinophils, macrophages, and mast cells. There is characteristic infiltration by lymphocytes and plasma cells deep in lamina propria (basal lymphoplasmacytosis). Crohns disease also shows evidence of crypt shortfall (do not reach muscularis mucosae), lymphoid aggregates may surround epithelial crypts - Normal follicles would push crypts peripherally and also criptitis and crypt abscess(Less common and less numerous than in UC). Another important feature of crohns disease is presence of inflammatory pseudopolyps. They are residual patches of mucosa between ulcerated areas.A point which favours towards a diagnosis of crohns disease is evidence of aphthous ulcers which are welldelineated, small lesions amidst normal mucosa often overlying and surrounding lymphoid aggregate. These ulcers are smaller with narrower base than in UC. They may coalesce to form larger, visible ulcer or deep fissure which can extend into submucosa, muscularispropria lined by plump histiocytes, foreign body giant cells. 
Crohns disease also shows transmural lymphoid aggregates, may have germinal centers, admixed plasma cells mostly in mucosa-submucosa junction and subserosa. Muscle hypertrophy (muscularis mucosae and propria), increase in ganglion cells in neuralplexus (neuromuscular hamartomas can also be formed) and fibrosis is also noted in crohnsdisease. Granulomas are formed in 40-60\% of CD resections, $25-40 \%$ of $\mathrm{CD}$ biopsies and are typically amidst lymphoplasmacytic inflammation. They are wellformed epithelioid granulomas (sarcoid-like) non necrotizing, with giant cells, lymphocytic rim mostly in submucosa and subserosa ${ }^{[13]}$.

Prompt recognition of toxic colitis is crucial because the process may lead to bowel perforation, peritonitis and death if not treated rapidly. The incidence of perforation of toxic colitis in a combined adult inflammatory bowel disease population was found to be high (17-46\%) [14]. This is important as perforation has been shown to be the most significant predictor of outcome, with mortality as high as $44 \%$ vs. $2 \%$ without perforation ${ }^{[15]}$. A further predictor of poor outcome is delay in surgery.

Toxic colitis occurs when the mucosal barrier and immune system are compromised, allowing bacteria to translocate through the walls of the colon and produce systemic symptoms of sepsis ${ }^{[16]}$. The colonic dilatation is thought to be mediated by both the inhibitory effect of nitric oxide and the inflammation-mediated destruction of myenteric and Auerbach plexuses, reducing colonic muscle tone ${ }^{[17]}$. Although initially described as 'toxic megacolon,' it is clear that marked dilation of the colon only occurs in a subset of these patients, and is not necessary for the process to progress to sepsis or perforation. Therefore, it is very important to identify the problem based on clinical and laboratory findings and not on the presence or absence of colonic dilatation.

The initial treatment of TM in patients with CD should be medical, including intravenous corticosteroids and broad-spectrum antibiotics.
Subtotal colectomy is recommended when distension persists or if improvement is not observed on maximal medical therapy after 24-72 h. Early surgery in patients without evidence of perforation results in lower mortality $(2-8 \%)$ than colectomy done after colic perforation has occurred $(40 \%)^{[4]}$.

Plain abdominal radiography is crucial for the diagnosis as well as for the day-to-day monitoring of patients with TM. The transverse or right colon is usually the most dilated colonic segment, frequently more than $6 \mathrm{~cm}$ and sometimes up to $15 \mathrm{~cm}$ on supine films. Early detection of TM is of clinical importance, mainly because surgery is mandatory in $50 \%$ of patients with severe acute colitis $^{[18]}$.

\section{Conclusion}

Toxic megacolon in crohn's disease is a serious and life threatening complication. There is utmost importance of early diagnosis of TM and rapid surgical intervention if improvement is not observed on medical therapy.

\section{References}

1. Marshak RH, Lester LJ. Megacolon a complication of ulcerative colitis. Gastroenterology 1950;16:768-72.

2. McMullen TP, Bailey RJ. Advances in the diagnosis and management of toxic megacolon. Can J Gastroenterol 2005;19: 163-4.

3. Moulin V, Dellon $\mathrm{P}$, Laurent $\mathrm{O}$, et al. Toxic megacolon in patients with severe acute colitis: computed tomographic features. Clin Imaging 2011; 35:431-36.

4. Sheth SG, LaMont JT. Toxic megacolon. Lancet 1998;351:509-13.

5. Makharia GK, Ramakrishna BS, Abraham $\mathrm{P}$, et al; Indian Society of Gastroenterology Task Force on Inflammatory Bowel Disease: Survey of inflammatory bowel diseases in India. Indian $\mathbf{J}$ Gastroenterol 2012;31:299-306. 
6. Platell C, Mackay J, Collopy B, et al. Anal pathology in patients with Crohn's disease. Aust N Z J Surg. 1996;66:5-9.

7. Stange EF, Travis SP, Vermeire S, et al. European evidence based consensus on the diagnosis and management of Crohn's disease: definitions and diagnosis. Gut. 2006;55:1-15.

8. H. Schachter, M.J. Goldstein, J.B. Kirsner. Toxic dilation complicating Crohn's disease of the colon. Gastroenterology, 53 (1967), pp. 136-142.

9. T. Tsuchiya, T. Watanabe, T. Konishi, H. Nagawa. Toxic megacolon associated with Crohn's disease. Gastrointest Endosc, 64 (2006), pp. 1012-1013.

10. K.N. Jalan, W. Sircus, W.I. Card, C.W. Fal coner, C.B. Bruce, G.P. Crean, et al. An experience of ulcerative colitis. Toxic dilation in 55 cases. Gastroenterology, 1 (1969), pp. 68-82.

11. J.H. Jones, M. Chapman. Definition of megacolon in colitis. Gut, 10 (1969), pp. 562-564.

12. D. Stewart, A. Chao, I. Kodner, E. Birnbau m, J. Fleshman, D. Dietz. Subtotal colectomy for toxic and fulminant colitis in the era of immunosuppressive therapy. Colorectal Dis, 11 (2009), pp. 184-190.

13. Diagnostic Pathology: Gastrointestinal, Second Edition, 1600 John F. Kennedy Blvd. Ste 1800 Philadelphia, PA 191032899, Elsevier, 2016, part V, section 1, 371.

14. A. Cunsolo, R.B. Bragaglia, N. Arena, M. G. Mastromatteo, R. Vecchi, G. Gozzetti.T oxic megacolon complicating ulcerative colitis and Crohn's disease. Int Surg, 70 (1985), pp. 339-343.
15. A.J. Greenstein, D.B. Sachar, A. Gibas, D. Schrag, T. Heimann, H.D. Janowitz, et al. Outcome of toxic dilatation in ulcerative and Crohn's colitis. J Clin Gastroenterol, 7 (1985), pp. 137-143.

16. D.C. Sabiston, M. Courtney, J.M.D. Town send, D. Beauchamp, M. Evers, K.L. Matt oxSabiston textbook of surgery Saunders (2008).

17. E.J.M. van Geenen, D.B. Sachar. Infliximab in Crohn's disease-associated toxic megacolon. J Clin Gastroenterol, 46 (2012), pp. 321-323.

18. Sheikh RA, Yasmeen S, Prindiville T. Toxic megacolon: a review. J K Practitioner2003;10:176-8. 